\title{
ARTICLE
}

\section{Nanoscale size effects in crystallization of metallic glass nanorods}

Sungwoo Sohn ${ }^{1, \star}$, Yeonwoong Jung ${ }^{1,2, \star, \dagger}$, Yujun $\mathrm{Xie}^{1,2}$, Chinedum Osuji ${ }^{3}$, Jan Schroers ${ }^{1}$ \& Judy J. Cha ${ }^{1,2}$

\begin{abstract}
Atomistic understanding of crystallization in solids is incomplete due to the lack of appropriate materials and direct experimental tools. Metallic glasses possess simple metallic bonds and slow crystallization kinetics, making them suitable to study crystallization. Here, we investigate crystallization of metallic glass-forming liquids by in-situ heating metallic glass nanorods inside a transmission electron microscope. We unveil that the crystallization kinetics is affected by the nanorod diameter. With decreasing diameters, crystallization temperature decreases initially, exhibiting a minimum at a certain diameter, and then rapidly increases below that. This unusual crystallization kinetics is a consequence of multiple competing factors: increase in apparent viscosity, reduced nucleation probability and enhanced heterogeneous nucleation. The first two are verified by slowed grain growth and scatter in crystallization temperature with decreasing diameters. Our findings provide insight into relevant length scales in crystallization of supercooled metallic glasses, thus offering accurate processing conditions for predictable metallic glass nanomolding.
\end{abstract}

\footnotetext{
${ }^{1}$ Department of Mechanical Engineering and Materials Science, Yale University, New Haven, Connecticut 06511, USA. ${ }^{2}$ Energy Sciences Institute, Yale West Campus, West Haven, Connecticut 06516, USA. ${ }^{3}$ Department of Chemical \& Environmental Engineering, Yale University, New Haven, Connecticut 06511, USA. * These authors contributed equally to this work. †Present address: Nanoscience Technology Center, Orlando, Florida 32826, USA. Correspondence and requests for materials should be addressed to J.J.C. (email: judy.cha@yale.edu).
} 
$\mathrm{N}$ anoscale metallic glasses (MGs) offer novel technological applications ${ }^{1}$ due to their superior mechanical properties such as high mechanical strength, high elasticity, high fracture toughness and high corrosion resistance ${ }^{2-9}$. In some cases, their unique chemical compositions are favourable for catalysis ${ }^{10}$, bioimplants ${ }^{11}$ and antibacterial applications $s^{12}$. Owing to the absence of an intrinsic length scale, hierarchical structures with multiscale surface features from centimeters down to nanometers are possible for MGs to engineer novel material properties ${ }^{10,13}$, which is challenging in crystalline metals. Patterning of MGs is typically achieved by thermoplastic forming ${ }^{14}$-pressing heated MGs into molds, where processing temperatures are in the supercooled liquid region bounded by the glass transition temperature and crystallization temperature. Most readily, molding is realized at the highest temperature where crystallization can still be avoided, thus retaining the attractive properties of the amorphous material ${ }^{15,16}$. For reliable thermoplastic processing of multiscale structuring of MGs, crystallization kinetics at all length scales must be understood. On the macroscale, crystallization kinetics have been determined for a wide range of alloys, enabling predictable molding ${ }^{15}$. By contrast, the crystallization behaviour of MG-forming liquids at nanoscale dimensions has not been carefully considered. This is in part due to the non-trivial experimental difficulty associated with detecting and accurately recording the heat signals from nanoscale samples as they undergo crystallization in the differential scanning calorimetry experiments that are typically used, to good effect, for macroscale samples. Despite experiments on a large ensemble of nanoscale samples indicating accelerated crystallization ${ }^{17}$, it is unclear whether crystallization kinetics remains unaltered at nanoscale. Up to now, macroscale processing parameters have been used for nanoscale molding of MGs with often-unpredictable crystallization behaviours ${ }^{18}$. Recently, nanocalorimetry has been used to study nanoscale samples ${ }^{19,20}$. One major challenge of nanocalorimetry is accuracy, and calorimetry alone cannot directly relate heat release with nanoscale MG morphology. In contrast, in-situ transmission electron microscope (TEM) techniques can simultaneously introduce and visualize structural changes to reveal phenomena that cannot be observed from ex-situ, ensemble techniques. In-situ TEM has been used to observe the dynamic structural changes of nanomaterials, such as growth of nanocrystals ${ }^{21}$ and nanowires ${ }^{22}$, and phase transformations in glassy materials ${ }^{23,24}$, providing a near atomistic understanding of their underlying mechanisms.

Here, we apply in-situ heating of MG nanorods inside a TEM and visualize their crystallization characteristics at unprecedentedly small length scales. We directly observe the structural changes of MG nanorods during crystallization and reveal unusual intrinsic size effects on their crystallization temperature, marking a departure from continuous flow description of MG kinetics.

\section{Results}

In-situ TEM heating. Amorphous $\mathrm{MG}$ nanorods of $\mathrm{Pt}_{57.5}$ $\mathrm{Cu}_{14.7} \mathrm{Ni}_{5.3} \mathrm{P}_{22.5}$ are prepared by a nanomolding technique ${ }^{14}$ (Supplementary Fig. 1). Figure 1a shows a representative TEM image of as-pressed MG nanorods on an in-situ TEM thermal grid that can be heated up to $1100^{\circ} \mathrm{C}$. The nanorods are heated from room temperature to above their liquidus temperature inside the TEM, and their structural changes during heating are observed using bright-field (BF) imaging, dark-field (DF) imaging and selective area electron diffraction (SAED) (Fig. 1b,c). The glass transition $\left(T_{\mathrm{g}}\right)$, crystallization $\left(T_{\mathrm{c}}\right)$ and melting $\left(T_{\mathrm{m}}\right)$ temperatures of bulk samples are indicated in Fig. $1 \mathrm{~b}$.
Size-dependent crystallization temperature. We characterize the crystallization behaviour of individual nanorods in the diameter range of $\sim 6-330 \mathrm{~nm}$ under in-situ heating. $T_{\mathrm{c}}$ is detected from SAED or DF TEM movies during heating (Fig. 2) as the onset of a rapid increase in the electron intensity in the back focal plane (SAED) or image plane (DF), respectively. SAED in-situ movies reveal that a thinner nanorod crystallizes at a lower $T_{c}$ than a thicker rod (Fig. 2a,b, Supplementary Movies 1 and 2). The general trend is a monotonically decreasing $T_{\mathrm{c}}$ with decreasing nanorod diameter down to $\sim 25 \mathrm{~nm}$. For nanorods below $25 \mathrm{~nm}, T_{\mathrm{c}}$ shows a reverse trend, increasing rapidly with decreasing nanorod diameter. Figure $2 \mathrm{c}$ shows snapshot BF TEM images that compare the crystallization kinetics of two nanorods with diameters below $\sim 25 \mathrm{~nm}$. Here, a thinner nanorod crystallizes at a higher $T_{\mathrm{c}}$ than a thicker one, which is the opposite behaviour of the ones observed in Fig. 2a,b. This reversed trend of $T_{\mathrm{c}}$ is best visible in tapered nanorods with narrowing diameters. Figure 2d shows snapshot DF TEM images of a tapered nanorod under in-situ heating (Supplementary Movie 3). Crystalline grains grow first in the thicker region and subsequently grow towards the thinner region. Remarkably, the thinner region, which is below $5 \mathrm{~nm}$, never crystallizes. Crystallization of an $\sim 6$-nm nanorod occurs at $\sim 340^{\circ} \mathrm{C}$ (Fig. 2e), which is $\sim 100{ }^{\circ} \mathrm{C}$ higher than the $T_{\mathrm{c}}$ for a $150-\mathrm{nm}$ nanorod (Fig. 2a). Figure $2 \mathrm{f}$ presents quantitatively measured $T_{c}$ 's of nanorods of various diameters under in-situ heating (see Methods). It reveals the initial decrease of $T_{c}$ with decreasing nanorod diameter and a rapid increase of $T_{c}$ below $25 \mathrm{~nm}$. No detectable changes in the chemical composition of nanorods were observed during in-situ heating by energy-dispersive X-ray spectroscopy (Supplementary Fig. 2) and cyclic heating experiments (Supplementary Fig. 3), ruling out chemical and electron beam effects as the origin for the observed $T_{\mathrm{c}}$ change.

A summary of $T_{c}$ 's for all considered nanorods is given in Fig. 3a. $T_{c}$ monotonically decreases with decreasing diameter down to $\sim 25 \mathrm{~nm}$, below which it rapidly increases. This nonmonotonic crystallization behaviour is discussed in the context of size-dependent apparent viscosity, nucleation probability and heterogeneous nucleation, all of which affect nucleation, growth rates and crystallization kinetics (Fig. 3b-e). For nanorods, heterogeneous nucleation on the surface of nanorods dominates over homogeneous nucleation, which would accelerate crystallization kinetics with decreasing nanorod diameter, thus lowering the $T_{\mathrm{c}}$ (Fig. 3b, black curve) ${ }^{25}$. Below a critical nanorod diameter, however, two additional factors affect the crystallization kinetics: reduced probability of nucleation and increase in the apparent viscosity.

\section{Discussion}

We discuss the increase in the apparent viscosity first. When the sample size approaches the size scale of intrinsic flow units such that the shear transformation zone can no longer freely operate, the flow resistance increases, causing apparent viscosity, $\eta$, to increase $\mathrm{e}^{26}$. Such departure from a continuum flow description, reflected in the increase of viscosity with decreasing confinement sizes, or equally, increasing Knudsen number, has been observed in other viscoelastic systems, including polymers or glassy materials ${ }^{26}$. The increase in viscosity would lead to decrease in nucleation rate and grain growth rate, as the jumping frequency and the diffusion constant are inversely proportional to the viscosity (Fig. 3b,c, red curves) ${ }^{27}$. We have experimentally investigated the growth kinetics in a nanorod with a locally narrowed region (Fig. 4). Indeed, we observe a suppressed grain growth rate in the narrower region during in-situ crystallization (Supplementary Movie 4). The growth front significantly slows 

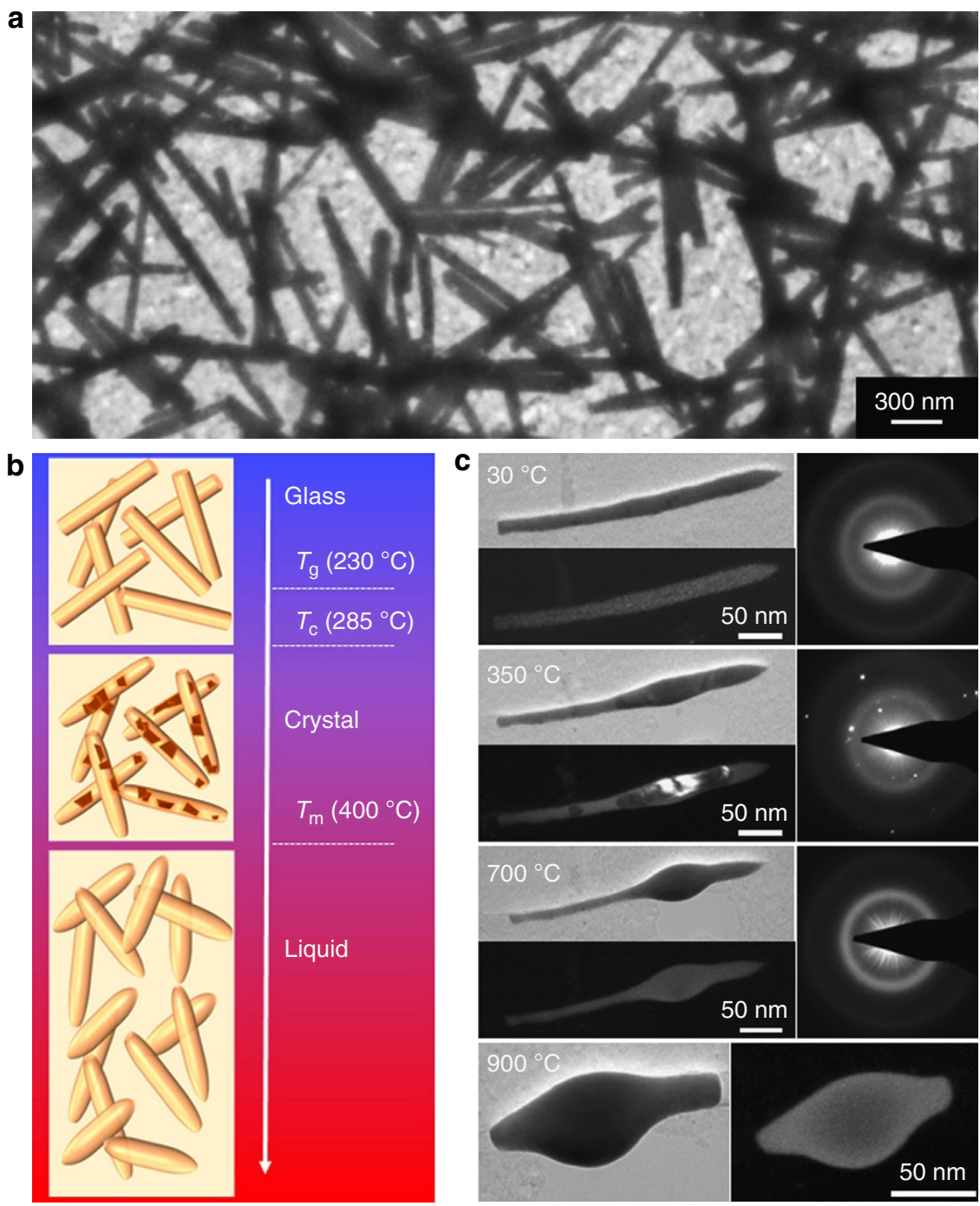

Figure 1 | Phase transition of MG nanorods under in-situ TEM. (a) TEM image of MG nanorods pressed into a nanomold with 55-nm-diameter holes. They are drop casts on a thin ceramic film, which gets heated inside TEM. (b) Schematics for the morphological change of MG nanorods at different stages of in-situ heating. As-pressed nanorods exhibit uniform TEM image contrast (first panel). As temperature increases above crystallization temperature, $T_{\mathrm{c}}$ crystalline grains start to grow in MG nanorods (dark brown in the second panel). The grains are identified by TEM imaging or electron diffraction. As temperature increases above the melting temperature, $T_{m}$, the crystallized nanorods turn to liquid phase (third panel). (c) TEM characterizations of a nanorod under in-situ heating. At $30^{\circ} \mathrm{C}$, the nanorod is amorphous, confirmed by the uniform TEM image contrast (top left: BF image, bottom left: DF image) and a diffusive ring pattern in SAED (right panel). Above $T_{\mathrm{c}}$ at $350^{\circ} \mathrm{C}$ the nanorod is largely crystallized, reflected in contrast changes in the TEM images. The crystalline phase appears dark for BF (top left) and bright for DF (bottom left) TEM images, respectively. The corresponding SAED displays diffraction spots, indicating the presence of crystalline grains (right panel). At $700^{\circ} \mathrm{C}$, which is above $T_{\mathrm{m}}$, uniform TEM image contrast and diffusive SAED ring similar to those at $30^{\circ} \mathrm{C}$ are observed to indicate that the nanorod is back to disordered state. As heating progresses with longer time, the nanorod becomes significantly deformed. At $900^{\circ} \mathrm{C}$, the nanorod becomes greatly shortened and balled-up.

down through the narrow region, as indicated by the snapshot DF TEM images (Fig. 4). The growth mode appears to remain the same throughout the entire growth time. Thus, this is a direct consequence of the increase in the apparent viscosity, which leads to the slowdown of crystallization kinetics and the increase of $T_{c}$, in agreement with our observation. Systematic size-dependent grain growth rate data are shown in Supplementary Fig. 4. Discussion on the growth mechanism can be found in Supplementary (Supplementary Figs 4,5).

In addition to the increasing apparent viscosity and heterogeneous nucleation, reduced probability of nucleation critically affects the crystallization kinetics, particularly for small nanorods. As the number of nuclei scales with the surface area of the nanorod, the probability of nucleation is anticipated to decrease if the nanorod size approaches the mean distance between nuclei (Fig. 3d, Supplementary Fig. 6). This ultimately slows down the crystallization kinetics, reflected as increase in $T_{c}$. Due to the stochastic nature of nucleation, the reduced probability of nucleation will result in a larger scatter in $T_{\mathrm{c}}$ for smaller rods. To confirm this, we performed in-situ crystallization experiments repeatedly and measured the scatter in $T_{\mathrm{c}}$ as a function of nanorod diameter. In agreement with our prediction, the scatter in $T_{\mathrm{c}}$ gradually increases for smaller nanorods (Fig. 5), pointing to the effect of the reduced probability of nucleation for small rods. The non-monotonic behaviour of $T_{c}$ shown in Fig. 3a, the main finding of this work, still holds even in the presence of the scatter. We note that a decrease in melting temperature $\left(T_{\mathrm{m}}\right)$, expected from the 

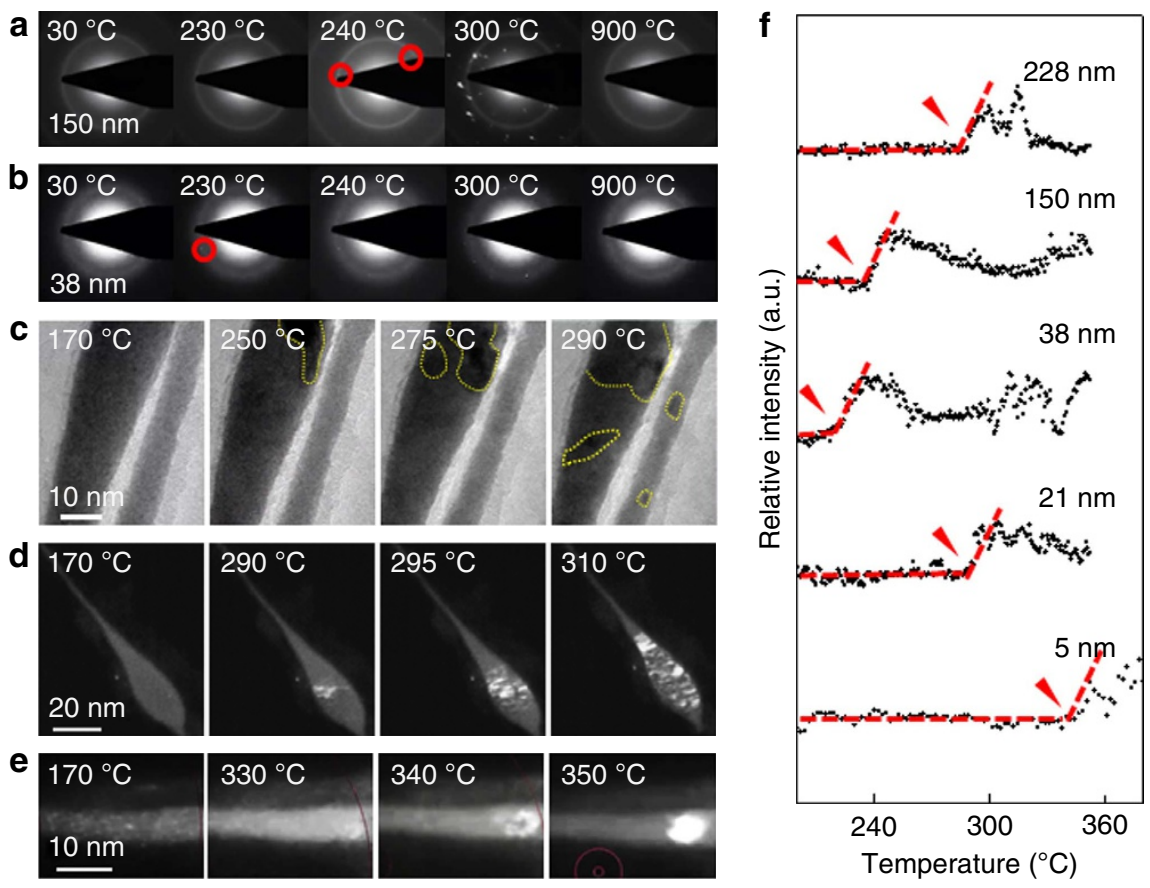

Figure 2 | In-situ characterization of the $\boldsymbol{T}_{\mathbf{c}}$ of nanorods with varying diameters. (a-e) Individual nanorods were heated inside the TEM at a constant ramping rate of $0.67^{\circ} \mathrm{Cs}^{-1}$ and their crystallization kinetics were monitored in real time. SAED and TEM imaging are used to identify the crystallization kinetics and $T_{\mathrm{c}}$. For thicker nanorods (diameter $>\sim 35 \mathrm{~nm}$ ), $T_{\mathrm{c}}$ was determined by identifying the onset of the first SAED spot. For smaller nanorods (diameter $<\sim 35 \mathrm{~nm}$ ), $T_{\mathrm{c}}$ was determined by identifying the TEM image contrast change in BF and DF modes. (a,b) Snapshot SAED to compare the crystallization kinetics of nanorods of 150 and $38 \mathrm{~nm}$ diameters, respectively. The red circles indicate the onset of the first diffraction spot, reflecting the onset of crystallization. (c) Snapshot BF TEM images to compare the crystallization kinetics of nanorods of diameters $<25 \mathrm{~nm}$. The dotted yellow lines indicate crystalline grains, which form at a lower temperature for the thicker nanorod. (d) Snapshot DF TEM images to show the crystallization kinetics of a tapered nanorod, revealing that the formation of crystalline grains (bright contrast) initiates on the thicker region. (e) Snapshot DF TEM images to show the crystallization kinetics of an extremely thin, $\sim 6$-nm-diameter nanorod, showing that crystalline grains form at $\sim 340^{\circ} \mathrm{C}$, which is over $50^{\circ} \mathrm{C}$ higher than the $T_{\mathrm{c}}$ of bulk MGs. (f) Quantification of $T_{\mathrm{c}}{ }^{\prime} \mathrm{s}$ for nanorods of various diameters based on the intensity change of TEM image contrasts. The abrupt increase of the relative intensity in each trace (denoted by red arrows) reflects the advent of first crystalline grains, which is defined as $T_{\mathrm{c}}$ (see Methods). The decrease of the intensity after $T_{\mathrm{c}}$ is attributed to the dynamic motion of grains under heating, so initial diffraction spots do not last.
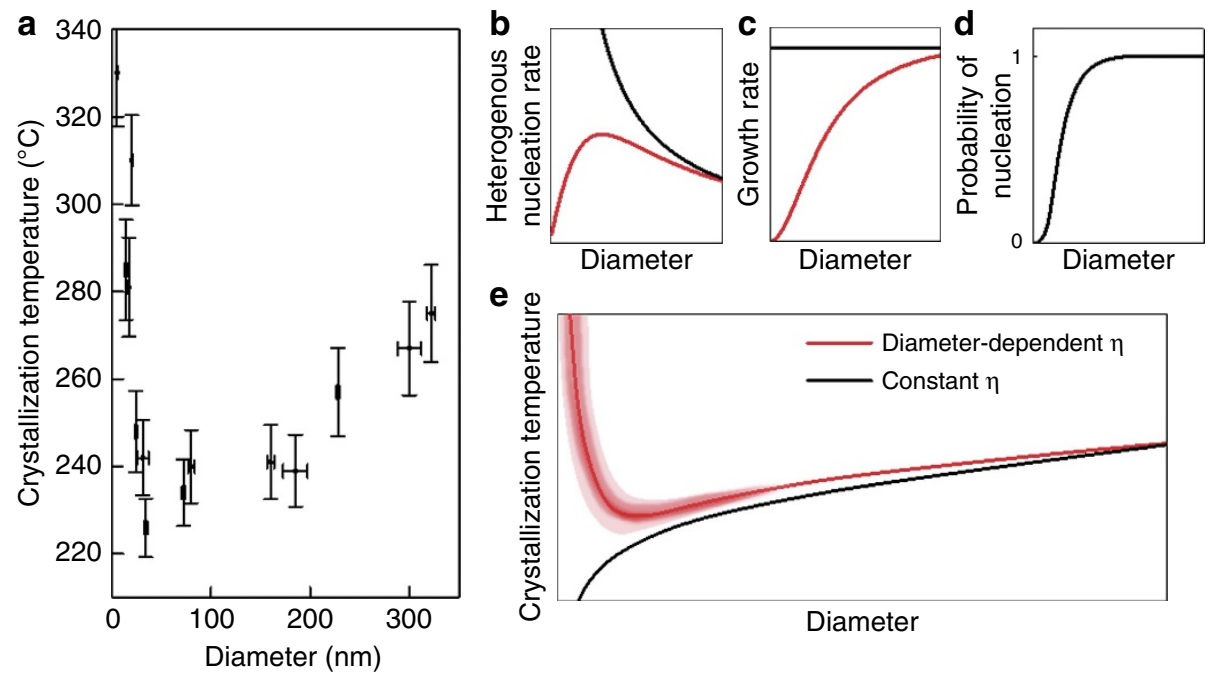

Figure 3 | Diameter-dependent crystallization kinetics of MG nanorods. (a) Correlation of $T_{\mathrm{c}}$ vs. nanorod diameter. All data were collected from non-tapered nanorods of uniform diameters. The uncertainty in $T_{\mathrm{c}}$ is calculated from the standard deviation of the temperature fluctuations during heating. (b-d) Schematics to illustrate the effects of surface-induced heterogeneous nucleation (b), apparent viscosity (c) and reduced nucleation probability (d) on crystallization kinetics. Red curves show the combined effect of the size-dependent surface-induced heterogeneous nucleation and apparent viscosity. The combined effect results in a size-dependent transition of the heterogeneous nucleation rate, while the growth rate monotonically decreases with decreasing diameter. This is due to the fact that diffusivity and the jumping frequency are inversely proportional to the viscosity. Black curves in $\mathbf{b}$ and $\mathbf{c}$ consider only the surface-enhanced nucleation. (e) The combined effect of size-dependent nucleation and growth rates results in a minimum of $T_{\mathrm{c}}$ at a certain diameter. The shaded area in red indicates the scatter in $T_{c}$ 's, reflecting the stochastic nature of nucleation at the reduced nucleation probability. 

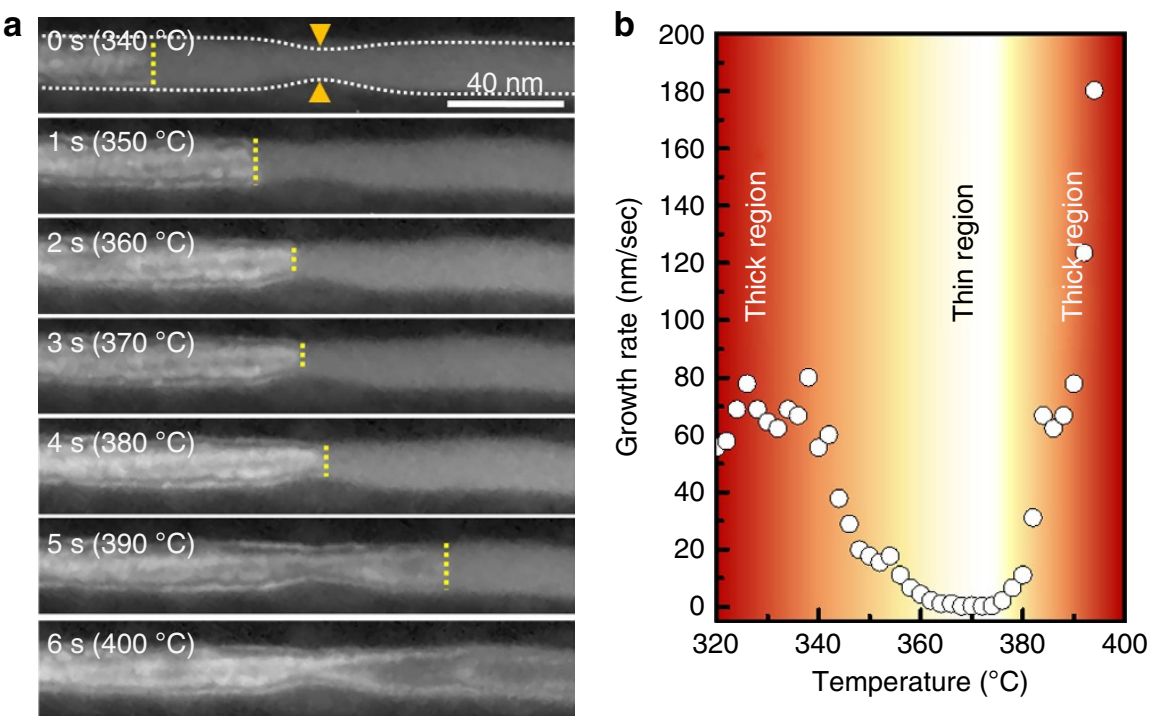

Figure 4 | Direct verification of growth rate difference in a nanorod. (a) Snapshot DF TEM images to observe the growth kinetics in a nanorod of $\sim 17$ nm with a narrow region ( $\sim 9 \mathrm{~nm}$, denoted by orange arrows) in diameter. The dotted line in the top image outlines the nanorod morphology. The grain growth rate is estimated by measuring the lateral expansion of the crystalline grain front, reflected as the change of imaging contrast (yellow dashed lines). Note that the growth front passes through the neck at $\sim 370^{\circ} \mathrm{C}$. (b) Quantification of the crystalline grain growth rate. The grain growth rate is suppressed at the narrow region of the nanorod during crystallization. The growth rate for the thicker region is $\sim 65$ and $\sim 180 \mathrm{~nm} \mathrm{~s}^{-1}$ at 330 and $394{ }^{\circ} \mathrm{C}$, respectively, and the rate for the thinner region is $4.5,0.3$ and $11 \mathrm{~nm} \mathrm{~s}^{-1}$, at 360,370 and $380^{\circ} \mathrm{C}$, respectively. The detailed experimental condition is described in Supplementary Note 2.
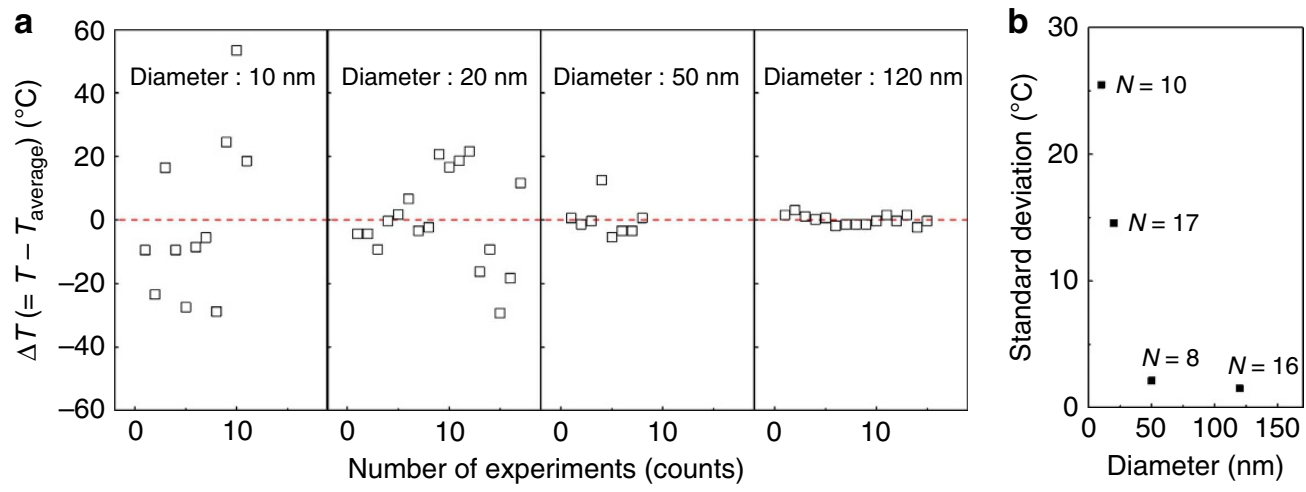

Figure 5 | Statistical studies on $\boldsymbol{T}_{\mathbf{c}}$ 's as a function of nanorod diameter. (a) The measured temperature scatter $\left(\Delta T=T-T_{\text {average }}\right.$ ), which is the difference between the crystallization temperatures $\left(T_{\mathrm{c}}\right)$ and the average $T_{\mathrm{c}}\left(T_{\text {average }}\right)$, and $(\mathbf{b})$ the corrected sample standard deviation of nanorods with a diameter of $10,20,50$ and $120 \mathrm{~nm}$, obtained from $N$ number of nanorods. The scatter increases with decreasing nanorod diameter, suggesting that the reduced probability of nucleation plays a critical role.

Gibbs-Thomson effect, does not alter the observed diameterdependent $T_{\mathrm{c}}$ (Supplementary Fig. 7).

Size-dependent $T_{\mathrm{c}}$ that we map out for nanoscale MGs down to $\sim 5 \mathrm{~nm}$ lays the foundation for their stable processing. The increase of $T_{c}$, owing to the reduced probability of nucleation, points to a larger processing window than previously assumed for feature sizes smaller than $\sim 30 \mathrm{~nm}$ to circumvent crystallization. By employing higher processing temperatures, current size limits for nanoscale MGs may be overcome. Fundamentally, our in-situ study of size effects on the crystallization behaviour of MGs reveals relevant length scales and a departure from a continuous flow description of MGs at the nanoscale.

\section{Methods}

Preparation of MG nanorods. The synthesis of $\mathrm{Pt}_{57.5} \mathrm{Cu}_{14.7} \mathrm{Ni}_{5.3} \mathrm{P}_{22.5} \mathrm{MG}$ nanorods used in this study is described in detail in our previous paper ${ }^{14}$. Briefly, alloying of high-purity constituents with nominal compositions was carried out in a vacuum-sealed quartz tube using induction melting. Subsequently, the master alloys were fluxed with boron trioxide $\left(\mathrm{B}_{2} \mathrm{O}_{3}\right)$ and vitrified by water quenching from a temperature of $1100^{\circ} \mathrm{C}$, which is $350^{\circ} \mathrm{C}$ above the thermodynamic liquidus temperature. A commercially available porous anodized aluminium oxide (AAO) mold (Synkera Inc.) was placed between two custom-built heating plates, at a constant temperature of $260^{\circ} \mathrm{C}$ (Supplementary Fig. 1a). A piece of bulk MG was placed and equilibrated on the AAO mold at $260^{\circ} \mathrm{C}$ for $30 \mathrm{~s}$ (Supplementary Fig. 1b). Nanorods were thermoplastically formed by pressing the bulk MG into the selected AAO mold, under a load at a linear ramping from 0 to $100 \mathrm{kN}$ (Supplementary Fig. 1c). The pressed sample was subsequently released from the mold by dissolving the AAO mold in a $20 \mathrm{wt} . \%$ potassium hydroxide $(\mathrm{KOH})$ solution, heated at $80^{\circ} \mathrm{C}$ for $\sim 10 \mathrm{~h}$ (Supplementary Fig. 1d). The released MG plate with nanorods attached was rinsed with distilled water and isopropyl alcohol (IPA). The rinsing process was repeated more than four times to minimize the residual salts and AAO mold fragments. Then, the nanorods were released and collected by sonication in IPA (Supplementary Fig. 1e). The minimum diameter of the AAO molds used was $13 \mathrm{~nm}$. Nanorods of diameters $<\sim 10 \mathrm{~nm}$ were stochastically produced in response to the dewetting process, by rapidly melting/ quenching as-pressed nanorods inside the TEM column $\left(\sim 10^{-5} \mathrm{~Pa}\right.$ vacuum level). The melt/quench was carried out at $900^{\circ} \mathrm{C}$ with a fast quenching rate of $\sim 10^{6} \mathrm{~K} \mathrm{~s}^{-1}$. Energy-dispersive $\mathrm{X}$-ray spectroscopy characterizations

(Supplementary Fig. 2) show no detectable differences in the chemical composition of nanorods irrespective of diameters and in-situ heating processes. 
In-situ TEM heating experiments. All TEM experiments were performed with a FEI Tecnai Osiris $200 \mathrm{kV}$ TEM. As-pressed nanorods dispersed in IPA were transferred to in-situ TEM substrates, via a drop casting method. We used commercially available in-situ TEM heating grids (E-AHA) and a heating holder (Aduro 300DT System) manufactured by Protochips, Inc. The heating substrate contains a resistive ceramic membrane contacted with metal electrodes, which introduce Joule heating to the membrane. The ceramic membrane contains an array of holes, over which a thin carbon film is overlaid. Nanorods sitting on the amorphous carbon film were inspected for $T_{\mathrm{c}}$ identification, so there is no contribution of the ceramic on diffraction spots or TEM imaging. The temperature profile of each substrate was pre-calibrated by the manufacturer. For the identification of $T_{\mathrm{c}}$, nanorods were first rapidly heated to $170^{\circ} \mathrm{C}$ (below $T_{\mathrm{g}}$ ) within $0.1 \mathrm{~s}$. Subsequently, a slower heating with a ramp rate of $0.67^{\circ} \mathrm{C} \mathrm{s}^{-1}$ was applied from 170 to $350^{\circ} \mathrm{C}$. During the heating, nanorods were monitored in real time under active DF/BF TEM imaging modes or SAED modes, which were simultaneously video-recorded (Snagit software).

\section{Quantification of $\boldsymbol{T}_{c}$ based on the intensity change in TEM imaging contrast.} Quantification of $T_{c}$ for nanorods with varying diameters was carried out by a series of intensity profile measurements made over an image contrast variation. Image frames of the BF or DF TEM movies recorded during in-situ heating were aligned using an automated pixel-based plug-in application, TurboReg (Biomedical Imaging Group, Swiss Federal Institute of Technology, Lausanne, Switzerland $)^{28}$ in ImageJ software (National Institutes of Health, Bethesda, MD) ${ }^{29}$. After alignment, two regions of interest (ROIs) were selected: one on the bright region of the sample (for BF/DF image movies) or on the first diffraction ring (for SAED pattern movies) and the other on the background. Intensity counts from the two ROIs were extracted from all image frames as a function of imaging time. The relative intensity profile for each nanorod was calculated as,

$$
\text { Relative Intensity }=\frac{I_{\text {sample }}-I_{\text {background }}}{I_{\text {background }}},
$$

where $I_{\text {sample(background) }}$ is the intensity of ROI from the sample (background). At the onset of crystallization, the relative intensity increases rapidly (Fig. 2f). We define $T_{\mathrm{c}}$ at the intercept of the constant background and the linear fit to the increasing relative intensity.

\section{References}

1. Greer, A. L. Metallic glasses. Science 267, 1947-1953 (1995).

2. Johnson, W. L. Bulk glass-forming metallic alloys: science and technology. MRS Bull. 24, 42-56 (1999).

3. Wang, W. H., Dong, C. \& Shek, C. H. Bulk metallic glasses. Mater. Sci. Eng. $R$ 44, 45-89 (2004).

4. Ashby, M. F. \& Greer, A. L. Metallic glasses as structural materials. Scripta Mater. 54, 321-326 (2006).

5. Schuh, C. A., Hufnagel, T. C. \& Ramamurty, U. Mechanical behavior of amorphous alloys. Acta Mater. 55, 4067-4109 (2007).

6. Guo, H. et al. Tensile ductility and necking of metallic glass. Nat. Mater. 6, 735-739 (2007).

7. Jang, D. \& Greer, J. R. Transition from a strong-yet-brittle to a stronger-andductile state by size reduction of metallic glasses. Nat. Mater. 9, 215-219 (2010).

8. Tian, L. et al. Approaching the ideal elastic limit of metallic glasses. Nat. Commun. 3, 609 (2012).

9. Chen, M. Mechanical behavior of metallic glasses: microscopic understanding of strength and ductility. Annu. Rev. Mater. Res. 38, 445-469 (2008).

10. Sekol, R. C. et al. Bulk metallic glass micro fuel cell. Small 9, 2081-2085 (2013).

11. Zberg, B., Uggowitzer, P. J. \& Loffler, J. F. MgZnCa glasses without clinically observable hydrogen evolution for biodegradable implants. Nat. Mater. 8, 887-891 (2009).

12. Schroers, J., Kumar, G., Hodges, T., Chan, S. \& Kyriakides, T. Bulk metallic glasses for biomedical applications. JOM 61, 21-29 (2009).

13. Schroers, J. Processing of bulk metallic glass. Adv. Mater. 22, 1566-1597 (2010).

14. Kumar, G., Tang, H. X. \& Schroers, J. Nanomoulding with amorphous metals. Nature 457, 868-872 (2009).

15. Bryn Pitt, E., Kumar, G. \& Schroers, J. Temperature dependence of the thermoplastic formability in bulk metallic glasses. J. Appl. Phys. 110, 043518 (2011).
16. Johnson, W. L. et al. Beating crystallization in glass-forming metals by millisecond heating and processing. Science 332, 828-833 (2011).

17. Gopinadhan, M. et al. Finite size effects in the crystallization of a bulk metallic glass. Appl. Phys. Lett. 103, 111912 (2013).

18. Carmo, M. et al. Bulk metallic glass nanowire architecture for electrochemical applications. ACS Nano 5, 2979-2983 (2011)

19. Gregoire, J. M. et al. Combining combinatorial nanocalorimetry and X-ray diffraction techniques to study the effects of composition and quench rate on $\mathrm{Au}-\mathrm{Cu}-\mathrm{Si}$ metallic glasses. Scripta Mater. 66, 178-181 (2012).

20. McCluskey, P. J. \& Vlassak, J. J. Combinatorial nanocalorimetry. J. Mater. Res 25, 2086-2100 (2010).

21. Zheng, H. et al. Observation of single colloidal platinum nanocrystal growth trajectories. Science 324, 1309-1312 (2009).

22. Kodambaka, S., Tersoff, J., Reuter, M. C. \& Ross, F. M. Germanium nanowire growth below the eutectic temperature. Science 316, 729-732 (2007).

23. Zhong, L., Wang, J., Sheng, H., Zhang, Z. \& Mao, S. X. Formation of monatomic metallic glasses through ultrafast liquid quenching. Nature $\mathbf{5 1 2}$ 177-180 (2014).

24. Salinga, M. et al. Measurement of crystal growth velocity in a melt-quenched phase-change material. Nat. Commun. 4, 2371 (2013).

25. Li, T., Donadio, D., Ghiringhelli, L. M. \& Galli, G. Surface-induced crystallization in supercooled tetrahedral liquids. Nat. Mater. 8, 726-730 (2009).

26. Shao, Z. et al. Size-dependent viscosity in the super-cooled liquid state of a bulk metallic glass. Appl. Phys. Lett. 102, 221901 (2013).

27. Orava, J., Greer, A. L., Gholipour, B., Hewak, D. W. \& Smith, C. E. Characterization of supercooled liquid $\mathrm{Ge}_{2} \mathrm{Sb}_{2} \mathrm{Te}_{5}$ and its crystallization by ultrafast-heating calorimetry. Nat. Mater. 11, 279-283 (2012).

28. Thevenaz, P., Ruttimann, U. E. \& Unser, M. A pyramid approach to subpixel registration based on intensity. IEEE Trans. Image Process. 7, 27-41 (1998).

29. Schneider, C. A., Rasband, W. S. \& Eliceiri, K.W. NIH Image to ImageJ: 25 years of image analysis. Nat. Methods 9, 671-675 (2012).

\section{Acknowledgements}

This work was supported by National Science Foundation MRSEC DMR 1119826. Microscopy facilities used in this work were supported by the Yale Institute for Nanoscience and Quantum Engineering (YINQE) and MRSEC DMR 1119826 .

\section{Author contributions}

S.S. and Y.J. equally contributed to the work. S.S., Y.J. and Y.X. carried out the in-situ TEM experiments under the direction of C.O., J.S. and J.J.C. S.S. performed the nanomolding experiments under the direction of J.S. S.S., Y.J. and J.J.C. analysed the TEM data. All authors (S.S., Y.J., Y.X., C.O., J.S. and J.J.C.) contributed to the discussion on the results and writing of the manuscript.

\section{Additional information}

Supplementary Information accompanies this paper at http://www.nature.com/ naturecommunications

Competing financial interests: The authors declare no competing financial interests

Reprints and permission information is available online at http://npg.nature.com/ reprintsandpermissions/

How to cite this article: Sohn, S. et al. Nanoscale Size Effects in Crystallization of Metallic Glass Nanorods. Nat. Commun. 6:8157 doi: 10.1038/ncomms9157 (2015).

This work is licensed under a Creative Commons Attribution 4.0 International License. The images or other third party material in this article are included in the article's Creative Commons license, unless indicated otherwise in the credit line; if the material is not included under the Creative Commons license, users will need to obtain permission from the license holder to reproduce the material. To view a copy of this license, visit http://creativecommons.org/licenses/by/4.0/ 